\title{
Aves acuáticas de la cuenca baja del río Sinú, Córdoba, Caribe Colombiano
}

\section{Waterbirds of the lower basin of the Sinú river, Colombian Caribbean}

\author{
Carlos Ruiz-Guerra ${ }^{\circ} \square$, Yanira Cifuentes-Sarmiento ${ }^{\circ} \square$
}

\section{Resumen}

Estudiamos la avifauna acuática del bajo Sinú, en el Caribe colombiano, entre 2009 y 2016. Se establecieron tres estaciones de muestreo por cada una de las cinco principales unidades de paisaje: playas, manglares, salitrales, humedales salobres y humedales dulceacuícolas. En cada una de las estaciones se evaluaron la abundancia, riqueza y diversidad de aves acuáticas, mediante censos semestrales. Se registraron 107 especies de aves acuáticas, de las cuales 14 corresponden a nuevos registros para el bajo Sinú. Los humedales dulceacuícolas albergaron la mayor riqueza y abundancia de aves acuáticas, mientras que los humedales salobres presentaron la mayor diversidad. De lo siete grupos funcionales de aves acuáticas, las vadeadoras presentaron las mayores concentraciones de individuos. Este estudio ratifica la importancia del bajo Sinú para la conservación de las aves del Caribe colombiano, pues los humedales de esta ecorregión ofrecen áreas de alimentación y de reproducción para cinco especies amenazadas.

Palabras clave. Avifauna. Estuario. Humedales. Manglar.

\begin{abstract}
We studied the waterbirds occurring at the lower basin of the Sinú River, in the Colombian Caribbean, from 2009 to 2016. We established three sampling stations in the five main landscape units of the lower Sinú: beaches, mangrove forests, salt flats, brackish marshes and freshwater wetlands. The abundance, richness and diversity of waterbirds were assessed in every station twice yearly. We recorded 107 species of waterbirds, including 14 new species records for that site. Freshwater wetlands held the highest species richness and abundance of waterbirds in the basin, whereas the brackish marshes contained the highest diversity. Wading birds were the functional group with the largest concentrations of individuals. This study confirms the importance of the lower basin of the Sinú River for birds in the Caribbean coast of Colombia, as its wetlands hold feeding and breeding sites for five threatened waterbirds species.
\end{abstract}

Key words. Avifauna. Estuary. Mangrove. Wetlands. 


\section{Introducción}

Los humedales proveen recursos alimenticios, sitios de reproducción y hábitat para una alta diversidad de aves migratorias y residentes (Elliott et al., 2020). Sin embargo, aún existen grandes vacíos de conocimiento sobre la historia natural de las aves neotropicales, que impiden entender las respuestas de las especies a los cambios ambientales (Lees et al., 2020). Por consiguiente, es necesaria una mayor cantidad de estudios sobre este grupo de vertebrados, para tener un conocimiento más profundo sobre la ecología, evolución y patrones de distribución de la biodiversidad en países del Neotrópico, como Colombia (Antonelli et al., 2018).

Con aproximadamente $27 \%$ de su territorio continental cubierto por humedales (Flórez et al., 2016), Colombia ha experimentado una notable transformación del paisaje, debido principalmente a la expansión de las áreas urbanas, en las que reside alrededor del $77 \%$ de la población. La población residente en las zonas costeras e insulares en el año 2019 era cercana a los 6300000 habitantes, de los cuales cerca del $87 \%$ se encontraba en la región Caribe. En esta región, en particular, el cambio en el uso del suelo ha ocasionado la degradación de grandes complejos de humedales, lo cual a su vez ha reducido su capacidad de brindar diferentes servicios ecosistémicos (Ricaurte et al., 2017).

El Caribe colombiano, localizado en el sector más septentrional de Suramérica, en su extremo noroccidental, tiene una zona costera emergida de $7673 \mathrm{~km}^{2}$, en los que se destacan tres deltas, correspondientes a los ríos Atrato, Sinú y Magdalena (Ospino et al., 2020). La cuenca baja del río Sinú, también conocida como bajo Sinú, está ubicada en el departamento de Córdoba y se destaca por albergar el bosque de mangle mejor conservado del Caribe colombiano (Sánchez-Páez et al., 2005). Esta cuenca incluye dos Áreas Importantes para la Conservación de las Aves y la biodiversidad (AICA): la Zona Deltaica Estuarina del Río Sinú y el Complejo Cenagoso de la Margen Occidental del Río Sinú (Franco \& Bravo, 2005); comprende asimismo dos áreas protegidas, bajo jurisdicción de la Corporación Autónoma Regional de los Valles del Sinú y San Jorge (CVS), que corresponden al Distrito Regional de Manejo Integrado Manglar de la Bahía de Cispatá y sector aledaño del Delta Estuarino del río Sinú y el Distrito Regional de Manejo Integrado Complejo Cenagoso del río Sinú (CVS \& Invemar, 2010).

La avifauna del bajo Sinú está conformada por 320 especies, pertenecientes a 62 familias, de las cuales 119 especies corresponden a aves acuáticas (Estela et al., 2010). En este artículo describimos la riqueza, abundancia y diversidad de las especies de aves acuáticas registradas entre 2009 y 2016 en el bajo Sinú. Así mismo, damos a conocer los registros de especies de aves acuáticas adicionales a los señalados por Estela \& López-Victoria (2005) y Estela et al. (2010) para esta cuenca, e incluimos evidencia reproductiva para algunas especies de aves.

\section{Materiales y métodos}

Área de estudio. El río Sinú, con $380 \mathrm{~km}$ de largo, nace en el Nudo de Paramillo, en el municipio de Ituango, departamento de Antioquia, a 3500 m s.n.m. y desemboca en el delta de Tinajones a través de tres bocas, denominadas Mireya, Medio y Correa, ubicadas en el municipio de San Bernardo del Viento, al sur del Golfo de Morrosquillo (Wilches et al., 2020). La cuenca baja del río Sinú, cubre un área aproximada de 150000 hectáreas y alberga el $80 \%$ de los humedales anexos al río Sinú (CVS, 2008). El área de estudio está localizada entre $9^{\circ} 18^{\prime}$ y $9^{\circ} 27^{\prime}$ latitud norte y entre $75^{\circ} 47^{\prime}$ y $75^{\circ} 59^{\prime}$ longitud oeste, en el departamento de Córdoba, Colombia (Figura 1). El régimen climático de la zona es moderadamente unimodal, con un periodo seco que inicia a comienzos de diciembre y se prolonga hasta marzo o abril, cuando las lluvias no sobrepasan los $50 \mathrm{~mm}$ al mes (Barreto et al., 1999). De esta manera, se puede diferenciar una época de lluvias entre agosto y diciembre y una época seca entre diciembre y abril (Barreto et al., 1999).

En 2009, subdividimos el área de estudio en cinco unidades de paisaje: playas, caños de manglar, salitrales, humedales salobres y humedales dulceacuícolas; para un total de 15 estaciones (Figura 1), nombradas de acuerdo con el conocimiento de pescadores y campesinos de la cuenca. Las estaciones de playa, denominadas Mestizo, Terraplén y Honda correspondieron a zonas abiertas con presencia de árboles de mangle y restos de esta vegetación. Las estaciones de caños de manglar, Ostional, Dago y La Muerte, se caracterizaron por presentar canales angostos de menos de $5 \mathrm{~m}$ de ancho y en medio de árboles de mangle. Los salitrales Nisperal, Bucanero y Cogepato, correspondieron a áreas abiertas, con aguas poco profundas y con plántulas y arbustos de mangle. En los humedales salobres de Sicará, Caño Grande y La Coroza predominaban islas de mangle, vegetación flotante, emergente y cultivos de arroz. Y finalmente los humedales dulceacuícolas de El Bongo, Isla Grande y Korea presentaban cultivos de arroz, árboles altos y arbustos propios de formaciones xerofíticas. 


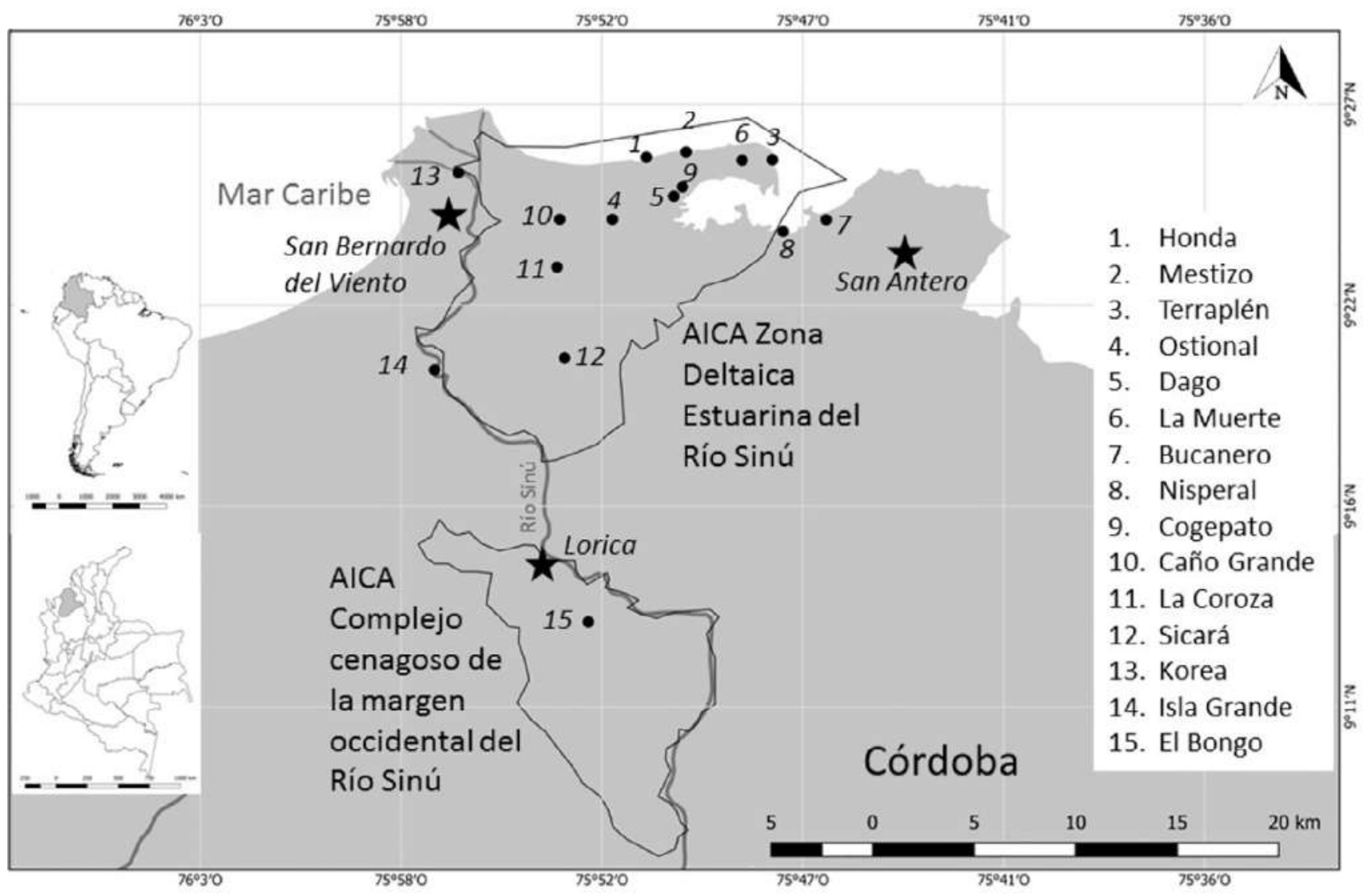

Figura 1. Ubicación geográfica de las 15 estaciones para muestreo de aves acuáticas en la cuenca baja del río Sinú, Caribe Colombiano.

Figure 1. Geographical location of the 15 sampling stations of waterbirds at the lower basin of the Sinú River, Colombian Caribbean.

Métodos. En cada una de las estaciones de muestreo ubicamos ocho puntos de radio fijo de $25 \mathrm{~m}$, separados 100 $\mathrm{m}$ entre sí, de acuerdo con los métodos propuestos por Ruiz-Guerra et al. (2011). En cada uno de los puntos, y durante cinco minutos, incluimos tanto los registros visuales como auditivos de las especies de aves acuáticas. Las visitas a cada estación se hicieron entre las 06:00 y las 10:00 horas, una vez por semestre entre 2009 y 2016. Adicionalmente, registramos evidencias directas de reproducción, tales como nidos con huevos o polluelos, adultos en los nidos, adultos que transportaban material de construcción y cópulas. No hicimos búsqueda intensiva de nidos, así que únicamente se incluye evidencia reproductiva observada en las estaciones de muestreo o en áreas cercanas. La abundancia se consideró como el número máximo de individuos obtenido en las 16 visitas realizadas; del mismo modo, consideramos a una especie como rara si estaba presente en menos del $5 \%$ de las 208 muestras y común si superaba ese porcentaje.
Clasificamos las especies registradas en siete grupos funcionales para su análisis: vadeadoras, limícolas, marinas y martinetes, patos y zambullidores, paludícolas, rapaces acuáticas y acuáticas no estrictas, según lo establecido por Ruiz-Guerra et al. (2014). El orden filogenético y la nomenclatura usados siguen la clasificación del South American Classification Committee; los nombres en español fueron tomados de Hilty \& Brown (2001); las categorías de amenaza a escala global, de acuerdo con la Unión Internacional para la Conservación de la Naturaleza mientras que la categorización a escala nacional sigue a Renjifo et al. (2016). Para las categorías de migración se consideraron las propuestas en Naranjo et al. (2012), con algunos ajustes. Adicionalmente, se revisó la base de datos del Laboratorio de Anillamiento de aves de Norteamérica (BBL) del USGS Patuxent Wildlife Research Center, para buscar información sobre aves acuáticas marcadas con anillos, que hubieran sido recapturadas en el bajo Sinú y reportadas al BBL. Utilizamos 
el índice de Margalef y el índice de Jaccard para el análisis de diversidad y similitud, respectivamente.

\section{Resultados}

Entre 2009 y 2016 registramos 107 especies de aves acuáticas, de las cuales 14 corresponden a adiciones a la lista de aves publicada por Estela \& López-Victoria (2005). Estas especies pertenecen a trece órdenes y 32 familias, e incluyen 59 especies migratorias: 33 invernantes no reproductivas, 21 invernantes con poblaciones reproductivas y cinco migratorios locales. Registramos 42 especies raras, de las cuales el $52 \%$ corresponde a aves residentes, mientras que el $57 \%$ de las aves comunes corresponden a especies migratorias (Anexo 1). Las aves vadeadoras y las limícolas presentaron el mayor número de especies, con 25 para cada grupo; las marinas y martinetes incluyeron 24 especies; las aves paludícolas, los patos y zambullidores y el grupo de acuáticas no estrictas incluyeron nueve especies cada uno; mientras que las rapaces acuáticas únicamente incluyeron seis especies (Tabla 1).

En la avifauna acuática del bajo Sinú se incluyen cuatro especies amenazadas en Colombia y una amenazada a escala global: la chavarría (Chauna chavaria), una especie de distribución restringida, que se encuentra bajo la categoría de vulnerable en Colombia y casi amenazada a escala global; la garza colorada (Agamia agami), especie vulnerable a escala global, cuya única colonia reproductiva conocida en el Caribe colombiano está ubicada en la región del bajo Sinú; la garza rojiza (Egretta rufescens), que está bajo las categorías de vulnerable en Colombia y casi amenazada globalmente; esta garza con poblaciones residentes y migratorias solo fue observada en su forma blanca en marzo y noviembre de 2011. Y, finalmente, el flamenco (Phoenicopterus ruber), que está en peligro en Colombia. Dos aves migratorias, el correlimos colorado (Calidris canutus) y el correlimos semipalmeado (Calidris pusilla), especies casi amenazadas a escala global, también hacen parte del grupo de aves acuáticas del bajo Sinú. La única especie de ave marina amenazada presente en esta cuenca es el piquero café (Sula leucogaster), que se encuentra en peligro en Colombia. Una de las especies asociadas al bosque de mangle en el bajo Sinú es el conirrostro manglero (Conirostrum bicolor), ave casi amenazada globalmente.

Con respecto a las 14 adiciones a la lista de especies, la mayoría corresponden a aves migratorias. En mayo de 2009, registramos en Nisperal nueve individuos del correlimos rabiblanco (Calidris fuscicollis) y un individuo muerto de petrel de Cory (Calonectris diomedea) (Ruiz-Guerra \& Cifuentes-Sarmiento, 2010); en noviembre de ese mismo año observamos en playa Mestizo un individuo del gaviotín enano (Sternula antillarum). En octubre de 2010 registramos en Isla Grande un individuo de correlimos zancón (Calidris himantopus), y en octubre de 2011, en Nisperal, un individuo de pato cucharo (Spatula clypeata). En mayo de 2012 observamos en Nisperal un individuo de flamenco ( $P$. ruber), mientras que en noviembre de ese mismo año registramos en Sicará un individuo de caracolero negro (Helicolestes hamatus). En mayo de 2013 adicionamos tres especies: un individuo de la polluela de antifaz (Porzana flaviventer) (Figura 2) en Sicará, un individuo de garzón soldado (Jabiru mycteria) en Korea y un individuo de caica común (Gallinago delicata). En mayo de 2015 registramos tres individuos de avetorillo bicolor (Ixobrychus exilis), dos en Sicará y uno en Korea. En septiembre de 2015 encontramos en Nisperal un individuo de tiñosa común
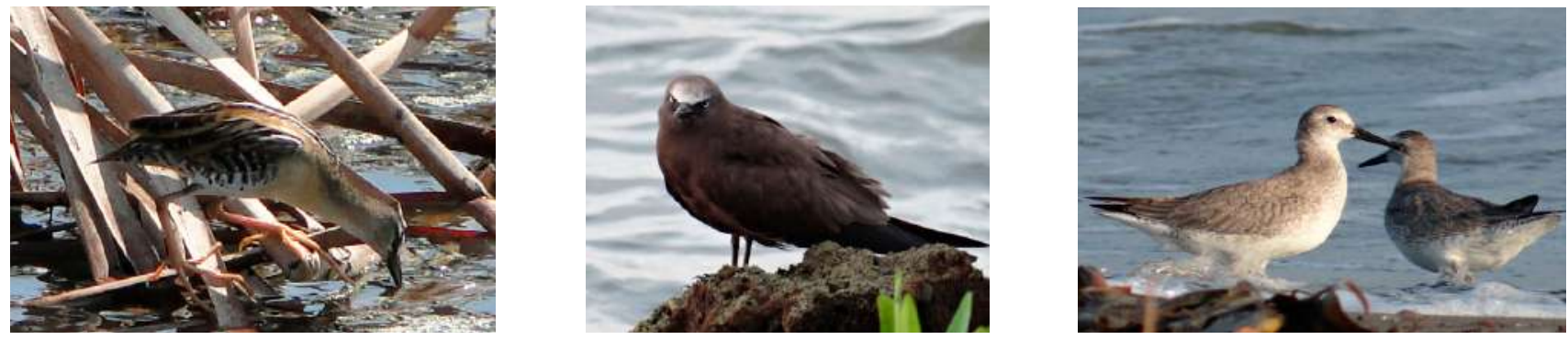

Figura 2. Algunas de las especies de aves acuáticas adicionadas entre 2009 y 2016 al bajo Sinú, Caribe colombiano. De izquierda a derecha: polluela de antifaz (Porzana flaviventer), tiñosa común (Anous stolidus) y correlimos colorado (Calidris canutus).

Figure 2. Some of the waterbirds added to the checklist of the lower Sinú River, Colombian Caribbean, between 2009 and 2016 . From left to right: Yellow-breasted Crake (Porzana flaviventer), Brown Noddy (Anous stolidus), and Red Knot (Calidris canutus). 
(Anous stolidus) y en playa Honda cuatro individuos del correlimos colorado (Calidris canutus) (Figura 2). Adicionalmente, en octubre de ese mismo año encontramos en Bongo un individuo muerto de polluela migratoria (Porzana carolina). A excepción del gaviotín enano (S. antillarum), el flamenco (P. ruber) y el correlimos zancón (C. himantopus), de los cuales obtuvimos registros en años posteriores, ninguna de las otras once especies fue registrada nuevamente (Anexo 2).

Abundancia, riqueza, diversidad y similitud. La mayor abundancia se encontró en los humedales dulceacuícolas y en los salitrales, mientras que los caños de manglar presentaron la menor abundancia. En cuanto a la riqueza, los humedales dulceacuícolas presentaron el mayor número de especies, seguidos por los caños de manglar y los salitrales. Por otro lado, los humedales salobres presentaron la mayor diversidad, seguidos por los humedales dulceacuícolas, mientras que los caños de manglar mostraron la menor diversidad (Tabla 1).

En cuanto a la similitud entre las unidades del paisaje, el índice de Jaccard indicó que las playas y los salitrales fueron las unidades de paisaje más similares entre sí, mientras que los humedales salobres y los caños de manglar presentaron la menor similitud de especies (Tabla 2).

Tabla 1. Abundancia (A), riqueza (R) y diversidad (D) de los grupos funcionales de aves acuáticas en cada una de las unidades del paisaje en el bajo Sinú, Caribe colombiano.

Table 1. Abundance (A), richness (R) and diversity (D) of functional groups of waterbirds in every landscape unit of the lower Sinu River, Colombian Caribbean.

\begin{tabular}{|c|c|c|c|c|c|c|c|c|c|c|c|c|c|c|c|}
\hline \multirow{2}{*}{$\begin{array}{c}\text { Grupos } \\
\text { funcionales }\end{array}$} & \multicolumn{3}{|c|}{$\begin{array}{l}\text { Humedales } \\
\text { dulceacuícolas }\end{array}$} & \multicolumn{3}{|c|}{$\begin{array}{l}\text { Caños de } \\
\text { manglar }\end{array}$} & \multicolumn{3}{|c|}{ Playas } & \multicolumn{3}{|c|}{ Salitrales } & \multicolumn{3}{|c|}{$\begin{array}{l}\text { Humedales } \\
\text { salobres }\end{array}$} \\
\hline & A & $\mathbf{R}$ & D & A & $\mathbf{R}$ & D & A & $\mathbf{R}$ & D & A & $\mathbf{R}$ & D & A & $\mathbf{R}$ & D \\
\hline Vadeadoras & 1958 & 19 & 2.37 & 371 & 15 & 2.37 & 268 & 16 & 2.68 & 3291 & 19 & 2.22 & 922 & 21 & 2.93 \\
\hline Limícolas & 512 & 18 & 2.73 & 11 & 3 & 0.83 & 529 & 19 & 2.87 & 572 & 21 & 3.15 & 198 & 10 & 1.70 \\
\hline Paludícolas & 189 & 6 & 0.95 & 4 & 1 & 0.00 & 0 & 0 & 0.00 & 6 & 2 & 0.56 & 110 & 6 & 1.06 \\
\hline Patos y zamb. & 1933 & 8 & 0.93 & 22 & 3 & 0.65 & 13 & 3 & 0.78 & 1218 & 6 & 0.70 & 1167 & 8 & 0.99 \\
\hline $\begin{array}{l}\text { Marinas y } \\
\text { martinetes }\end{array}$ & 1598 & 13 & 1.63 & 45 & 10 & 2.36 & 662 & 14 & 2.00 & 123 & 11 & 2.08 & 80 & 12 & 2.51 \\
\hline Rapaces & 26 & 5 & 1.23 & 9 & 4 & 1.37 & 9 & 2 & 0.46 & 7 & 3 & 1.03 & 18 & 4 & 1.04 \\
\hline No estrictas & 175 & 7 & 1.16 & 51 & 3 & 0.51 & 10 & 5 & 1.74 & 77 & 6 & 1.15 & 163 & 8 & 1.37 \\
\hline Total & 6391 & 76 & 8.56 & 513 & 39 & 6.09 & 1491 & 59 & 7.94 & 5294 & 68 & 7.81 & 2658 & 69 & 8.62 \\
\hline
\end{tabular}

Tabla 2. Similitud de especies (índice de Jaccard) de aves acuáticas entre unidades de paisaje en el bajo río Sinú, Caribe colombiano.

Table 2. Similarity of waterbirds species (Jaccard Index) between landscape units of the lower Sinu River, Colombian Caribbean.

\begin{tabular}{lccccc}
\hline \multicolumn{1}{c}{ Unidades del paisaje } & Humedal de río & Caños de manglar & Playas & Salitrales & Humedales estuarinos \\
\hline Humedales de río & 1 & & & & \\
\hline Caños de manglar & 0.29 & 1 & & & \\
\hline Playas & 0.26 & 0.32 & 1 & & \\
\hline Salitrales & 0.23 & 0.22 & 0.42 & 1 & 1 \\
\hline Humedales estuarinos & 0.2 & 0.15 & 0.22 & 0.38 & \\
\hline
\end{tabular}




\section{Grupos funcionales}

Vadeadoras: en este grupo, el ibis blanco (Eudocimus albus), la garza real (Ardea alba), la garza patiamarilla (Egretta thula) y el ibis pico de hoz (Plegadis falcinellus) fueron las especies más abundantes; la garza real (A. alba) presentó el mayor número de registros (Anexo 2). Las mayores concentraciones de individuos de vadeadoras fueron observadas en los salitrales, mientras que la menor, en las playas. Los humedales salobres presentaron la mayor riqueza y diversidad de vadeadoras (Tabla 1). Por otro lado, encontramos que la garza colorada (A. agami), la garza cucharón (Cochlearius cochlearius), el guaco común (Nycticorax nycticorax), la garcita del ganado (Bubulcus ibis), la garza real (A. alba), la garza tricolor (Egretta tricolor), la garza patiamarilla (E. thula) y el ibis blanco (E. albus) comparten una colonia de reproducción, localizada en La Coroza. En contraste, el vaco mexicano (Tigrisoma mexicanum), se reproduce de manera solitaria en el manglar. Observamos nidos del vaco mexicano ubicados de manera dispersa y a más de $5 \mathrm{~m}$ de altura en árboles de mangle rojo (Rhizophora mangle). Estos nidos presentaron polluelos en abril de 2012, mayo de 2013 y mayo de 2015 y adultos en el nido en octubre de 2009, marzo de 2011, junio de 2014 y julio de 2016 (Anexo 2). Por otra parte, aunque Egretta thula se considera como especie invernante con poblaciones reproductivas permanentes (Ruiz-Guerra, 2012), no se tenía evidencia de la presencia de individuos migratorios en el bajo Sinú, pero el reporte del BBL sobre un individuo de esta especie, anillado en 1969 en Virginia (Estados Unidos) y encontrado en 1975 en el bajo Sinú, así lo confirmó.

Limícolas: las especies más abundantes de este grupo fueron el correlimos picudo (Calidris mauri), el gallito de ciénaga (Jacana jacana) y la becasina piquicorta (Limnodromus griseus). En cuanto a número de registros, el andarríos maculado (Actitis macularius) presentó el mayor número, seguido por el gallito de ciénaga (J. jacana) y el pellar común (Vanellus chilensis) (Anexo 2). Los salitrales presentaron la mayor abundancia, riqueza y diversidad de limícolas (Tabla 1).

Encontramos que cinco especies de limícolas anidan en esta cuenca: el gallito de ciénaga (J. jacana), el pellar común (V. chilensis), el chorlito piquigrueso (Charadrius wilsonia), el chorlito collarejo (Charadrius collaris) y la cigüeñuela (Himantopus mexicanus). El gallito de ciénaga probablemente se reproduce todo el año en el bajo Sinú, pues observamos pollos de esta especie en diferentes ocasiones (Anexo 2). De igual forma, registramos varios pollos del pellar común en el primer semestre del año; sin embargo, ni del gallito de ciénaga ni del pellar común encontramos nidos con huevos. La reproducción del chorlito collarejo y de la cigüeñuela ya había sido documentada para el bajo Sinú (Cifuentes-Sarmiento \& Ruiz-Guerra, 2009; Ruiz-Guerra \& Cifuentes-Sarmiento, 2013). En cuanto al chorlito piquigrueso (C. wilsonia), en mayo de 2013 encontramos un nido con un huevo bajo el cuidado de dos parentales; posteriormente, en junio de 2014 hallamos una hembra que incubaba dos huevos y en mayo de 2015 observamos un pollo bajo el cuidado de un adulto (Figura 3); estos tres registros de reproducción fueron obtenidos en Playa Honda y corresponden a la primera evidencia de anidación de la especie en el departamento de Córdoba.

Marinas y martinetes: el cormorán neotropical (Phalacrocorax brasilianus) fue la especie con mayor número de individuos observados y el martín pescador
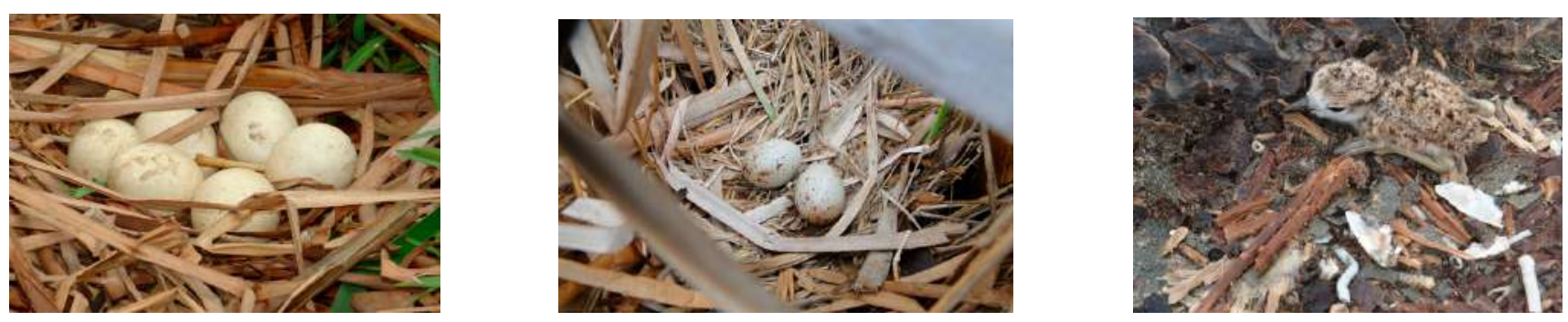

Figura 3. Evidencia reproductiva de algunas aves acuáticas en el bajo Sinú, Caribe colombiano. De izquierda a derecha: nido con huevos de chavarría (Chauna chavaria), nido con huevos de polla gris (Gallinula galeata) y polluelo de chorlito piquigrueso (Charadrius wilsonia cinnamominus).

Figure 3. Breeding evidence of waterbirds in the lower Sinu River, Colombian Caribbean. From left to right: nest with eggs of Northern Screamer (Chauna chavaria), nest with eggs of Common Moorhen (Gallinula galeata), and a nestling of Wilson's Plover (Charadrius wilsonia cinnamominus). 
mayor (Megaceryle torquata) fue la especie con mayor número de registros (Anexo 2). Los humedales dulceacuícolas presentaron la mayor abundancia de individuos de este grupo, mientras que la mayor riqueza se presentó en las playas y la mayor diversidad en los humedales salobres (Tabla 1). Obtuvimos evidencia directa de reproducción de tres especies de este grupo: para el cormorán neotropical (P. brasilianus) observamos nidos en octubre de 2015 y julio de 2016 en La Coroza; adicionalmente en octubre de 2010 se observaron en Korea dos individuos que transportaban material para construcción de nidos y en noviembre 2011, otros dos individuos con material en La Coroza. El único nido registrado de pato aguja (Anhinga anhinga) lo observamos en La Coroza en octubre de 2015. Del martín pescador matraquero (Chloroceryle amazona) encontramos un nido ubicado en un barranco en Caño Grande en 2010 y 2012, el nido fue reutilizado por dos años y su detección fue posible, gracias al constante ingreso de parentales con presas en el pico.

Para este grupo, el BBL suministró datos de tres gaviotines: un individuo de gaviotín piquirrojo (Hydroprogne caspia) recapturado en el bajo Sinú provino de la provincia canadiense de Ontario. Tres individuos de gaviotín real (Thalasseus maximus) provenían cada uno de tres estados de la región Noreste de Estados Unidos: Maryland, Carolina del Norte y Virginia. Y un individuo de gaviotín patinegro (Thalasseus sandvicensis), originario de Carolina del Norte, fue hallado en el bajo Sinú casi un año después de ser anillado en su área de reproducción en 1991.

Patos y zambullidores: la iguaza común (Dendrocygna autumnalis) fue la especie de este grupo con mayor número de registros y con los números más altos de individuos observados (Anexo 2). Los humedales dulceacuícolas presentaron la mayor abundancia y riqueza, mientras que la mayor diversidad se encontró en los humedales salobres (Tabla 1). La única evidencia de reproducción encontrada para representantes de este grupo correspondió a la iguaza común (D. autumnalis), de la cual observamos pollos. Otra especie que probablemente anida en el bajo Sinú, es el zambullidor chico (Tachybaptus dominicus), debido a que se observó un individuo en plumaje nupcial en noviembre de 2012 en Isla Grande, pero no fue considerado como una evidencia directa de reproducción en el área de estudio. De acuerdo con el BBL, se han recapturado individuos del pato careto (Spatula discors) en el bajo Sinú que provienen de Minnesota, Dakota del Sur y Dakota del Norte (Estados Unidos), así como de la provincia canadiense de Manitoba.
Paludícolas: en este grupo la chavarría ( $C$. chavaria) fue la especie más abundante, así como, el ave con mayor número de registros, seguida por el carrao (Aramus guarauna) (Anexo 2). La mayor abundancia de paludícolas fue encontrada en los humedales dulceacuícolas; la riqueza fue muy similar al resto de unidades del paisaje, con excepción de las playas, donde no se registró ningún paludícola, y los caños de manglar, donde apenas se registró una especie del grupo (Tabla 1). Encontramos evidencia de anidación de cinco de las ocho especies de aves paludícolas. En noviembre de 2012 registramos un nido en construcción de la chavarría (C. chavaria), en un eneal (Typha sp.) en Sicará y en octubre de 2015, detectamos un nido con seis huevos en un eneal cercano a un arrozal en Caño Grande. Por otro lado, en Sicará, en mayo de 2013, encontramos un nido de polla gris (Gallinula galeata) con dos huevos a punto de eclosionar (Figura 3). Posteriormente, en marzo de 2011, observamos dos pollos de esta ave en Sicará, y en diciembre de 2014 cinco polluelos bajo el cuidado de dos adultos en Isla Grande. Otra paludícola que anida en el bajo Sinú es la polla azul (Porphyrio martinicus), de la cual encontramos un nido con cuatro huevos en Caño Grande en mayo de 2009; así mismo, en noviembre de 2011 observamos dos polluelos con dos adultos de la polla azul en Caño Grande. En diciembre de 2013, registramos en Bongo tres adultos de esta paludícola, con un polluelo cada uno.

Rapaces acuáticas: en este grupo se destacaron el caracolero común (Rostrhamus sociabilis) como la especie más abundante y el cangrejero negro (Buteogallus anthracinus) como la rapaz con mayor número de registros (Anexo 2). La mayor abundancia y riqueza se encontraron en los humedales dulceacuícolas, mientras que la diversidad más alta de este grupo se encontró en los caños de manglar (Tabla 1). Las tres aves rapaces con evidencia reproductiva fueron el caracolero común (R. sociabilis), el cangrejero negro (B. anthracinus) y el cangrejero mayor (Buteogallus urubitinga) (Anexo 2). De acuerdo con el BBL, un individuo de águila pescadora (Pandion haliaetus) que fue encontrado en el manglar del bajo Sinú en julio de 2010 provenía de Connecticut, Estados Unidos.

Acuáticas no estrictas: en cuanto a las aves acuáticas no estrictas, el turpial cabeciamarillo (Chrysomus icterocephalus) fue la especie con mayor número de individuos de este grupo y la viudita común (Fluvicola pica) y el garrapatero mayor (Crotophaga major) fueron las especies con mayor número de registros (Anexo 2). Estas mismas tres especies fueron las únicas del grupo 
con evidencia reproductiva. Los humedales dulceacuícolas albergaron la mayor abundancia de este grupo, mientras que la mayor riqueza se presentó en los humedales salobres y la mayor diversidad en las playas (Tabla 1).

\section{Discusión}

De acuerdo con las colecciones ornitológicas que reposan en el Field Museum of Natural History de Chicago, varias localidades del bajo Sinú fueron visitadas entre 1916 y 1929 en búsqueda de especímenes de aves; sin embargo, el conocimiento de la avifauna de esta área del departamento de Córdoba permaneció por años escasamente estudiada hasta finales del siglo XX e inicios del siglo XXI, periodo en el que se publicaron los trabajos de Dahl \& Medem (1964), Naranjo (1979), de Ayala (1997), Ruiz-Ovalle (2002), Estela (2004), Estela et al. (2004), Estela et al. (2005), Estela \& López-Victoria (2005), Rodríguez-Barrios \& Troncoso (2006), Racero-Casarrubio et al. (2008), Ruiz-Guerra et al. (2008), Estela et al. (2010), Ruiz-Guerra \& Cifuentes-Sarmiento (2010, 2013), Burgos et al. (2014) y Ruiz-Guerra \& Echeverri-Galvis (2019). El bajo Sinú y la ecorregión Ciénaga Grande de Santa Marta son las únicas áreas del Caribe colombiano que cuentan con al menos diez publicaciones sobre aves acuáticas entre 2000 y 2020.

A pesar de los diversos estudios en la zona, el conocimiento de la avifauna acuática del bajo Sinú presenta vacíos relacionados con su estado de conservación; ejemplo de esto es la polluela bicolor (Laterallus exilis), la cual no ha sido registrada desde el año 2000 (Estela \& López-Victoria, 2005) y no es posible establecer si ha sufrido extinción local. Por otro lado, Racero-Casarrubio et al. (2008) sostuvieron que el garzón soldado (J. mycteria) había sido extirpado en el bajo Sinú, lo cual se descarta con este estudio, aunque sí es una especie rara en el área.

Por otra parte, la cuenca del bajo Sinú alberga cuatro especies amenazadas en Colombia y una amenazada a escala global, la garza colorada (A. agami), cuya única colonia conocida en el país está ubicada en La Coroza, por lo cual debe ser considerada una de las prioridades en las acciones de manejo y conservación del DRMI Manglar de la Bahía de Cispatá y sector aledaño del Delta Estuarino del río Sinú. Se debe también considerar que La Coroza es usada por otras aves acuáticas como sitio de anidación, por lo que la presencia de una especie invasora, la garcita del ganado (Bubulcus ibis), que inició a anidar allí en diciembre de 2014 podría tener un impacto no solo para la vegetación presente en la colonia, sino para las aves que allí se reproducen. La garza colorada en La Coroza debe enfrentar los retos propios de la competencia por recursos en una colonia reproductiva: a los frecuentes hallazgos de polluelos muertos o heridos se les pueden sumar aspectos como la depredación natural (en una ocasión observamos cómo un individuo del guaco común, Nycticorax nycticorax se alimentó de un polluelo de garza colorada) y el robo de material para la construcción de nidos por otras especies de vadeadoras.

De igual forma, en La Coroza se reproducen otras especies de aves acuáticas, como es el ibis blanco (Eudocimus albus) del cual observamos reproducción desde julio a noviembre. Según Estela y López-Victoria (2005), el ibis blanco anidaba en Agrosoledad; no obstante, durante este estudio no se encontró ningún tipo de evidencia de reproducción de esa especie en ese sector. Por otro lado, la única colonia de reproducción del ibis blanco que había sido reportada para el Caribe colombiano se encuentra en Isla Tesoro-Parque Nacional Natural Corales del Rosario y de San Bernardo (Duque-García \& Franke-Ante, 2011), ubicada a 90 $\mathrm{km}$ de La Coroza. Por lo tanto, actualmente estas dos son las únicas colonias reproductivas conocidas del ibis blanco para la región; sin embargo, y dada la cercanía, es necesario conocer la relación entre estas dos colonias reproductivas y otras áreas de congregación de individuos de aves acuáticas del bajo Sinú y otras localidades de la región Caribe.

Otro aspecto que se debe resaltar del bajo Sinú es el de representar un área de paso e invernada para aves migratorias, pues el 53 \% de su avifauna acuática corresponde a especies migratorias. De hecho, doce de las catorce especies adicionadas corresponden a aves acuáticas migratorias, mientras que solo siete especies migratorias registradas por Estela y López-Victoria (2005) no fueron encontradas por nosotros: el chorlo dorado (Pluvialis dominica), el chorlito colirrojo (Charadrius vociferus), la becasina piquilarga (Limnodromus scolopaceus), el falaropo tricolor (Phalaropus tricolor), el págalo pomarino (Stercorarius pomarinus), el págalo del Polo Sur (Stercorarius maccormicki) y el martín pescador migratorio (Megaceryle alcyon). En el bajo Sinú las mayores concentraciones de individuos de los grupos funcionales limícolas, marinas y martinetes, así como de patos y zambullidores, fueron conformadas principalmente por bandadas de especies migratorias, tales 
como el correlimos picudo (Calidris mauri), el gaviotín real (T. maximus) y el pato careto (S. discors). Por otro lado, aunque existe poca información en el BBL sobre aves recapturadas en el área de estudio, probablemente la mayoría de las especies de gaviotines migratorios que visitan el bajo Sinú provienen del corredor migratorio del Atlántico.

En lo que respecta a las amenazas que la avifauna acuática enfrenta en la cuenca baja del río Sinú, de acuerdo con la CVS (2004) en las partes baja y media de la cuenca, recursos como la flora y fauna han disminuido considerablemente por el avance de la frontera agrícola, pecuaria y por la intervención con obras civiles, tales como la Central Hidroeléctrica Urrá I, sobre la red de drenaje que intercomunicaba los humedales con los caños, las ciénagas y el río Sinú. Si se tiene en cuenta que aproximadamente el $80 \%$ de las aguas servidas de Montería, capital de Córdoba, y los municipios aledaños se vierten directamente a la cuenca del río Sinú (CVS, 2004) resulta necesario conocer como la contaminación del río Sinú ha afectado la avifauna y sus hábitats. Burgos et al. (2014) encontraron altas concentraciones de mercurio por encima de $5.0 \mathrm{mg} / \mathrm{kg}$ en plumas e hígado de individuos jóvenes y adultos del pelícano común (Pelecanus occidentalis) que fueron capturados en el bajo Sinú. Adicionalmente, Garcés-Ordóñez et al. (2020) evidenciaron la presencia de microplásticos en peces recolectados en el bajo Sinú, por lo que es probable que otras poblaciones de aves marinas y martinetes, vadeadoras y rapaces acuáticas puedan ser afectadas por mercurio y microplásticos.

Adicionalmente, aunque Racero-Casarrubio et al. (2008) señalaron que la cacería de aves es una actividad frecuente en el bajo Sinú, únicamente encontramos evidencia de una captura con redes de pesca de un individuo de vaco mexicano (T. mexicanum) y la presencia de algunos individuos en cautiverio de chavarría (C. chavaria). Probablemente, otras especies de aves terrestres son presa de cazadores y saqueadores de nidos para ser comercializadas, pero durante nuestras visitas al área de estudio no observamos este tipo actividades.

El complejo de humedales de la cuenca baja del río Sinú representa una oportunidad de conservación para las aves acuáticas del Caribe colombiano, al albergar 107 especies de aves, seis de las cuales están incluidas en la lista roja de UICN y cuatro en el Libro Rojo en Colombia. Tanto el bosque de mangle como los humedales de esta cuenca son claves, tanto para la avifauna como para las comunidades humanas que dependen en gran medida del recurso pesquero.

\section{Agradecimientos}

Agradecemos a INVEMAR y a la empresa Urrá S.A. por permitir llevar a cabo esta investigación en el bajo Sinú, en especial a Lina Ramos y a William Zubiría. Gracias a Felipe Estela, Robin Correa, Remberto de la Rosa, Domingo Rodríguez Julio, Lorena Cabeza y a la Asociación Calidris por apoyar esta investigación.

\section{Referencias}

Antonelli, A., Ariza, M., Albert, J., Andermann, T., Azevedo, J., Bacon, C., Faurby, S., Guedes, T., Hoorn, C., Lohmann, L.G., Matos-Maraví, P., Ritter, C.D., Sanmartín, I., Silvestro, D., Tejedor, M., ter Steege, H., Tuomisto, H., Werneck, F.P., Zizka, A. \& Edwards, S.V. (2018). Conceptual and empirical advances in Neotropical biodiversity research. PeerJ, 6, 1-53.

https:/ / doi.org/10.7717/ peerj.5644

Barreto M., Barrera R., Benavides, J., Cardozo, E., Hernández, H., Marín, L., Posada, B., Salvatierra, C., Sierra, P. \& Villa A. (1999). Diagnóstico ambiental del Golfo de Morrosquillo (Punta Rada, Tolú). Una aplicación de sensores remotos y SIG como contribución al manejo integrado de zonas costeras. (Informe técnico). Tolú: Instituto Geográfico Agustín Codazzi y Centro InterAmericano de Fotointerpretación. 207 pp.

Burgos, S., Marrugo, J. M., Navarro, A. \& Urango, I. (2014). Mercurio en Pelecanus occidentalis de la bahía de Cispatá, Colombia. Revista MVZ Córdoba, 19(2), 4168-4174.

https:/ / doi.org/10.21897/rmvz.110

Cifuentes-Sarmiento, Y. \& Ruiz-Guerra, C. (2009). Current status of Black-necked Stilt and Collared Plover neotropical Shorebirds overlooked in Colombia. Wader Study Group Bulletin, 116(2), 113-114.

CVS-Corporación Autónoma Regional de los Valles del Sinú y del San Jorge. (2004). Diagnóstico ambiental de la cuenca hidrográfica del río Canalete, delimitación, extensión, localización y situación ambiental. (Informe técnico). Montería: Corporación Autónoma Regional de los Valles del Sinú y San Jorge. 320 pp.

CVS-Corporación Autónoma Regional de los Valles del Sinú y del San Jorge. (2008). Plan de Gestión Ambiental Regional-PGAR actualización 2008-2019. (Informe técnico). Montería: Corporación Autónoma Regional de los Valles del Sinú y San Jorge. 268 pp.

CVS-Corporación Autónoma Regional de los Valles del Sinú y del San Jorge \& INVEMAR. (2010). Plan integral de manejo del Distrito de Manejo Integrado (DMI) bahía de Cispatá - La Balsa - Tinajones y sectores aledaños 
del delta estuarino del río Sinú, departamento de Córdoba. (Informe técnico). Santa Marta: INVEMAR.141 pp.

Dahl, G. \& Medem, F. (1964). Informe sobre la fauna acuática del río Sinú (Informe técnico). Bogotá D.C.: Corporación Autónoma Regional de los Valles Magdalena y Sinú. 109 pp.

de Ayala, R.M. (1997). Inventario de la avifauna de los manglares del Caribe colombiano. (Trabajo de grado). Cali: Universidad del Valle, Facultad de Ciencias Naturales y Exactas, Departamento de Biología. 68 pp.

Duque-García, D.L. \& Franke-Ante, R. (2011). Aves. En Zarza-González, E. (Ed.). El entorno ambiental del Parque Nacional Natural Corales del Rosario y de San Bernardo. Pp 246-260. Cartagena de Indias: Parques Nacionales Naturales de Colombia Dirección Territorial Caribe Parque Nacional Natural Corales del Rosario y de San Bernardo.

Elliott, L., Igl, L., \& Johnson, D. (2020). The relative importance of wetland area versus habitat heterogeneity for promoting species richness and abundance of wetland birds in the Prairie Pothole Region, USA. Condor, 122(1) 1-21.

https:/ / doi.org/10.1093/condor/duz060

Estela, F. (2004). Observaciones del págalo del Polo Sur (Catharacta maccormicki) en el Caribe colombiano. Ornitología Colombiana 2, 50-52.

Estela, F., Naranjo, L.G. \& Franke-Ante, R. (2004). Registros de págalos (aves: Stercorariidae) En las costas de Colombia. Boletín de Investigaciones Marinas y Costeras, 33, 245-250.

Estela, F., Jaramillo, J.G. \& Mejía-Tobón, A. (2005). Ampliación de distribución de la tortolita escamada (Columbina squammata) en el Caribe colombiano. Boletín SAO, 15, 105-110.

Estela, F. \& López-Victoria, M. (2005). Aves de la parte Baja del Río Sinú, Caribe colombiano; inventario y ampliaciones de distribución. Boletín de Investigaciones Marinas y Costeras, 34, 7-42.

Estela, F., Ruiz-Guerra, C., Solano, O. D \& Ortiz-Ruiz, J. (2010). Aves del estuario del río Sinú. Santa Marta: Invemar, Asociación Calidris y Empresa Urrá S.A.E.S.P. 39 pp.

Flórez, C., Estupiñán-Suárez, L.M., Rojas, S., Aponte, C., Quiñones, M., Acevedo, O., Vilardy, S. \& Jaramillo, U. (2016). Identificación espacial de los sistemas de humedales continentales de Colombia. Biota Colombiana, 17 (Suplemento 1 - Humedales), 44-62.

https:/ / doi.org/10.21068/c2016s01a03

Franco, A.M. \& Bravo, G. (2005). Áreas importantes para la conservación de las aves en Colombia. En BirdLife \&
Conservación International (Eds.). Áreas importantes para la conservación de las aves en los Andes tropicales: sitios prioritarios para la conservación de la biodiversidad. Pp:142-143. Quito: BirdLife International.

Garcés-Ordóñez, O., Mejía-Esquivia, K.A., Sierra-Labastidas, T., Patiño, A., Blandón, L.M. \& Espinosa Díaz, L. F. (2020). Prevalence of microplastic contamination in the digestive tract of fishes from mangrove ecosystem in Cispatá, Colombian Caribbean. Marine Pollution Bulletin 154, 1-7.

https:// doi.org/10.1016/j.marpolbul.2020.111085

Hilty, S. H. \& Brown, W.L. (2001). Guía de las aves de Colombia. Cali: Princeton University Press, American Bird Conservancy-ABC, Universidad Del Valle y Sociedad Antioqueña de Ornitología. 1030 pp.

Lees, A., Rosenberg, K., Ruiz-Gutiérrez, V., Marsden, S., Schulenberg, T. \& Rodewald, A. (2020). A roadmap to identifying and filling shortfalls in Neotropical ornithology. Auk, 137, 1-17.

https:/ / doi.org/10.1093/auk/ ukaa048

Naranjo, L. G. (1979). Las aves marinas del Caribe colombiano: taxonomía, zoogeografía y anotaciones ecológicas. (Trabajo de grado). Bogotá: Fundación Universidad Jorge Tadeo Lozano, Facultad de Biología Marina. $279 \mathrm{pp}$.

Naranjo, L.G., Amaya, J.D., Eusse-González, D. \& Cifuentes-Sarmiento, Y. (2012). Guía de las especies migratorias de la biodiversidad en Colombia. Bogotá D.C.: Ministerio de Ambiente y Desarrollo Sostenible y WWF Colombia. 708 pp.

Ospino Sepulveda, L. J., García Valencia, C., Romero, D.C., Pizarro Pertuz, J.J., Hernández Narváez, D.M. \& Zamora Bornachera, A.P. (2020). Los espacios oceánicos y zonas costeras e insulares de Colombia. 19-20. En: Invemar. Informe del estado de los ambientes y recursos marinos y costeros en Colombia, 2019. Serie de Publicaciones Periódicas No. 3. Santa Marta. 183 p.

Racero-Casarrubio, J. A., Ballesteros-Correa, J., Gil, N., Ruiz, O. \& Reyes, K. (2008). Avifauna asociada al complejo cenagoso del bajo Sinú, departamento de Córdoba, Colombia. Revista de la Asociación Colombiana de Ciencias Biológicas, 20, 59-73.

Renjifo, L. M., Amaya-Villarreal, A. M., Burbano-Girón, J. \& Velásquez-Tibatá, J. (2016). Libro rojo de aves de Colombia, Volumen II: Ecosistemas abiertos, secos, insulares, acuáticos continentales, marinos, tierras altas del Darién y Sierra Nevada de Santa Marta y bosques húmedos del centro, norte y oriente del país. Bogotá D. C.: Editorial Pontificia Universidad Javeriana e Instituto Alexander von Humboldt. 563 pp. 
Ricaurte, L. F., Olaya-Rodríguez, M. H., Cepeda-Valencia, J., Lara, D., Arroyave-Suárez, J., Finlayson, C. M., \& Palomo, I. (2017). Future impacts of drivers of change on wetland ecosystem services in Colombia. Global Environmental Change, 44, 158-169.

https:/ / doi.org/10.1016/j.gloenvcha.2017.04.001

Rodríguez-Barrios, J. \& Troncoso, F. (2006). Éxito de anidación de la garza real Egretta alba (aves, Ardeidae) en el departamento de Córdoba, Colombia. Acta Biológica Colombiana, 11, 111-121.

Ruiz-Guerra, C., Johnston-González, R., Castillo-Cortés, L.F., Cifuentes-Sarmiento, Y., Eusse-González, D. \& Estela, F. (2008). Atlas de Aves Playeras y otras Aves Acuáticas en la costa Caribe colombiana. Santiago de Cali: Asociación Calidris. 77 pp.

Ruiz-Guerra, C. \& Cifuentes-Sarmiento, Y. (2010). Primer registro del petrel de Cory (Calonectris diomedea) para Colombia. Ornitología Colombiana, 10, 65-68.

Ruiz-Guerra, C., Castillo, F., Cifuentes-Sarmiento, Y. Johnston-González, R. \& Zamudio, J. (2011). Manual para censos de aves acuáticas en hábitats costeros. Experiencia basada en el complejo Marino-Costero Iscuandé-Sanquianga-Gorgona. Santiago de Cali: Asociación Calidris. 53 pp.

Ruiz-Guerra, C. (2012). Egretta thula. 120-121. En: Naranjo, L. G., J. D. Amaya, D. Eusse-González \& Y. Cifuentes-Sarmiento (Eds). Guía de las Especies Migratorias de la Biodiversidad en Colombia. Pp 120121. Bogotá D.C.: Ministerio de Ambiente y Desarrollo Sostenible y WWF Colombia. Colombia.

Ruiz-Guerra, C. \& Cifuentes-Sarmiento, Y. (2013). Primeros registros de anidación del Chorlito Collarejo
(Charadrius collaris) en Colombia. Ornitología Colombiana, 13, 37-43.

Ruiz-Guerra, C., D. Eusse-González \& Arango, C. (2014). Distribución, abundancia y reproducción de las aves acuáticas de las sabanas inundables de Meta y Casanare (Colombia) y sitios prioritarios para la conservación. Biota Colombiana, 15, 137-160.

Ruiz-Guerra, C. \& Echeverry-Galvis, M.A. (2019). Prey consumed by wading birds in mangrove swamps of the Caribbean coast of Colombia. Journal of Natural History, 53, 1823-1836.

https:/ / doi.org/10.1080/00222933.2019.1667037

Ruiz-Ovalle, J. M. (2002). Uso y selección de sitios de percha en la interfase agua vegetación de tres hábitats asociados a aguas lénticas y lóticas por la avifauna del nororiente del Departamento de Córdoba. (Trabajo de grado). Bogotá D.C.: Pontificia Universidad Javeriana. $113 \mathrm{pp}$.

Sánchez-Páez, H., Ulloa-Delgado, G., Tavera-Escobar, H. \& Gil-Torres, W. (2005). Plan de manejo integral de los manglares de la zona de uso sostenible sector estuarino de la bahía de Cispatá departamento de Córdoba. Bogotá D.C.: Ministerio de Ambiente, Vivienda y Desarrollo Territorial. 202 pp.

Wilches, F. J., Feria Díaz, J.J. \& Hernández Ávila, J. (2020). Physicochemical characterization of the waters of the Sinú River, downstream of the Urrá reservoir, north of Colombia. International Journal of Engineering Research and Technology, 13(10), 29092914.

https:/ / doi.org/10.37624/IJERT/13.10.2020.2909-2914 


\section{Anexos}

Anexo 1. Lista de especies de aves acuáticas registradas entre 2009 y 2016 en el bajo río Sinú, Caribe colombiano. El asterisco corresponde a especies adicionadas a la lista de Estela \& López-Victoria (2005). Estatus migratorio: INR, invernante no reproductivo; IPR, invernante con poblaciones reproductivas; ML, migratoria local; R, residente. Frecuencia: c, común; r, raro.

Appendix 1. List of waterbirds species recorded from 2009 to 2016 in the lower Sinú River, Colombian Caribbean. The asterisk indicates the new birds added to Estela \& López-Victoria's (2005) list. Migratory status: INR, migrant without resident populations; IPR, migrant with resident populations; $\mathrm{ML}$, local migrant; $\mathrm{R}$, resident. Frequency: $\mathrm{c}$, common; r, rare.

\begin{tabular}{|c|c|c|c|c|c|}
\hline Orden & Familia & Especie & Nombre común & Estatus & Frecuencia \\
\hline \multirow{8}{*}{ Anseriformes } & Anhimidae & Chauna chavaria & Chavarría & ML & c \\
\hline & \multirow{7}{*}{ Anatidae } & Dendrocygna bicolor & Iguaza maría & $\mathrm{R}$ & c \\
\hline & & Dendrocygna viduata & Iguaza careta & $\mathrm{R}$ & c \\
\hline & & $\begin{array}{l}\text { Dendrocygna } \\
\text { autumnalis }\end{array}$ & Iguaza común & ML & C \\
\hline & & Cairina moschata & Pato real & $\mathrm{R}$ & $\mathrm{r}$ \\
\hline & & Spatula discors & Pato careto & IPR & c \\
\hline & & Spatula clypeata* & Pato cucharo & INR & $\mathrm{r}$ \\
\hline & & Nomonyx dominicus & Pato encapuchado & $\mathrm{R}$ & $\mathrm{r}$ \\
\hline \multirow{2}{*}{ Podicipediformes } & \multirow{2}{*}{ Podicipedidae } & Tachybaptus dominicus & Zambullidor chico & $\mathrm{R}$ & $\mathrm{r}$ \\
\hline & & Podilymbus podiceps & Zambullidor común & $\mathrm{R}$ & $\mathrm{r}$ \\
\hline Phoenicopteriformes & Phoenicopteridae & Phoenicopterus ruber* & Flamenco & ML & $\mathrm{r}$ \\
\hline Procellariiformes & Procellariidae & Calonectris diomedea* & Petrel de Cory & INR & $\mathrm{r}$ \\
\hline \multirow{2}{*}{ Ciconiiformes } & \multirow{2}{*}{ Ciconiidae } & Jabiru mycteria* & Garzón soldado & $\mathrm{R}$ & $\mathrm{r}$ \\
\hline & & Mycteria americana & Cabeza de hueso & $\mathrm{R}$ & r \\
\hline \multirow{4}{*}{ Suliformes } & Fregatidae & Fregata magnificens & Fragata común & $\mathrm{R}$ & c \\
\hline & Sulidae & Sula leucogaster & Piquero café & ML & $\mathrm{r}$ \\
\hline & Phalacrocoracidae & $\begin{array}{l}\text { Phalacrocorax } \\
\text { brasilianus }\end{array}$ & Cormorán neotropical & IPR & c \\
\hline & Anhingidae & Anhinga anhinga & Pato aguja & $\mathrm{R}$ & $\mathrm{r}$ \\
\hline \multirow{8}{*}{ Pelecaniformes } & Pelecanidae & Pelecanus occidentalis & Pelicano común & IPR & c \\
\hline & \multirow{7}{*}{ Ardeidae } & Tigrisoma lineatum & Vaco colorado & $\mathrm{R}$ & c \\
\hline & & Tigrisoma mexicanum & Vaco mexicano & $\mathrm{R}$ & c \\
\hline & & Agamia agami & Garza colorada & $\mathrm{R}$ & $\mathrm{r}$ \\
\hline & & Cochlearius cochlearius & Garza cucharón & $\mathrm{R}$ & $\mathrm{r}$ \\
\hline & & Ixobrychus exilis* & Avetorillo bicolor & $\mathrm{R}$ & $\mathrm{r}$ \\
\hline & & Nycticorax nycticorax & Guaco común & IPR & c \\
\hline & & Nyctanassa violacea & Guaco Manglero & IPR & c \\
\hline
\end{tabular}




\begin{tabular}{|c|c|c|c|c|c|}
\hline Orden & Familia & Especie & Nombre común & Estatus & Frecuencia \\
\hline \multirow{15}{*}{ Pelecaniformes } & \multirow{11}{*}{ Ardeidae } & Butorides virescens & Garcita verde & IPR & c \\
\hline & & Butorides striata & Garcita rayada & $\mathrm{R}$ & c \\
\hline & & Bubulcus ibis & Garcita del ganado & IPR & c \\
\hline & & Ardea herodias & Garzón migratorio & INR & c \\
\hline & & Ardea cocoi & Garzón azul & $\mathrm{R}$ & c \\
\hline & & Ardea alba & Garza real & IPR & c \\
\hline & & Pilherodius pileatus & Garza crestada & $\mathrm{R}$ & $\mathrm{r}$ \\
\hline & & Egretta tricolor & Garza tricolor & IPR & c \\
\hline & & Egretta rufescens & Garza rojiza & IPR & $\mathrm{r}$ \\
\hline & & Egretta thula & Garza patiamarilla & IPR & c \\
\hline & & Egretta caerulea & Garza azul & IPR & c \\
\hline & \multirow{4}{*}{ Threskiornithidae } & Eudocimus albus & Ibis blanco & IPR & c \\
\hline & & Plegadis falcinellus & Ibis pico de hoz & IPR & c \\
\hline & & Phimosus infuscatus & Coquito & $\mathrm{R}$ & c \\
\hline & & Platalea ajaja & Espátula & $\mathrm{R}$ & c \\
\hline \multirow{6}{*}{ Accipitriformes } & Pandionidae & Pandion haliaetus & Águila pescadora & INR & c \\
\hline & \multirow{5}{*}{ Accipitridae } & Rostrhamus sociabilis & Caracolero común & $\mathrm{R}$ & c \\
\hline & & Helicolestes hamatus* & Caracolero negro & $\mathrm{R}$ & $\mathrm{r}$ \\
\hline & & $\begin{array}{l}\text { Buteogallus } \\
\text { anthracinus }\end{array}$ & Cangrejero negro & $\mathrm{R}$ & c \\
\hline & & Buteogallus urubitinga & Cangrejero mayor & $\mathrm{R}$ & $\mathrm{r}$ \\
\hline & & Busarellus nigricollis & Águila cienaguera & $\mathrm{R}$ & c \\
\hline \multirow{8}{*}{ Gruiformes } & Aramidae & Aramus guarauna & Carrao & $\mathrm{R}$ & c \\
\hline & \multirow{7}{*}{ Rallidae } & Aramides cajaneus & Chilacoa colinegra & $\mathrm{R}$ & c \\
\hline & & Aramides axillaris & Chilacoa costera & $\mathrm{R}$ & $\mathrm{r}$ \\
\hline & & Laterallus albigularis & Polluela chocoana & $\mathrm{R}$ & $\mathrm{r}$ \\
\hline & & Porzana flaviventer* & Polluela de antifaz & $\mathrm{R}$ & $\mathrm{r}$ \\
\hline & & Porzana carolina* & Polluela migratoria & INR & $\mathrm{r}$ \\
\hline & & Gallinula galeata & Polla gris & IPR & c \\
\hline & & Porphyrio martinicus & Polla azul & $\mathrm{R}$ & c \\
\hline \multirow{5}{*}{ Charadriiformes } & \multirow{5}{*}{ Charadriidae } & Vanellus chilensis & Pellar común & $\mathrm{R}$ & c \\
\hline & & Pluvialis squatarola & Chorlo pechinegro & INR & c \\
\hline & & $\begin{array}{l}\text { Charadrius } \\
\text { semipalmatus }\end{array}$ & Chorlito semipalmeado & INR & c \\
\hline & & Charadrius wilsonia & Chorlito piquigrueso & IPR & c \\
\hline & & Charadrius collaris & Chorlito collarejo & IPR & c \\
\hline
\end{tabular}




\begin{tabular}{|c|c|c|c|c|c|}
\hline Orden & Familia & Especie & Nombre común & Estatus & Frecuencia \\
\hline \multirow{33}{*}{ Charadriiformes } & Recurvirostridae & Himantopus mexicanus & Cigüeñuela & IPR & C \\
\hline & \multirow{18}{*}{ Scolopacidae } & Gallinago delicata* & Caica común & INR & $\mathrm{r}$ \\
\hline & & Limnodromus griseus & Becasina piquicorta & INR & C \\
\hline & & Numenius phaeopus & Zarapito común & INR & c \\
\hline & & Actitis macularius & Andarríos maculado & INR & c \\
\hline & & Tringa melanoleuca & Andarríos mayor & INR & c \\
\hline & & Tringa flavipes & Andarríos patiamarillo & INR & c \\
\hline & & Tringa solitaria & Andarríos solitario & INR & c \\
\hline & & Tringa semipalmata & Andarríos alinegro & INR & c \\
\hline & & Arenaria interpres & Vuelvepiedras & INR & c \\
\hline & & Calidris canutus* & Correlimos colorado & INR & $\mathrm{r}$ \\
\hline & & Calidris alba & Correlimos blanco & INR & $\mathrm{r}$ \\
\hline & & Calidris pusilla & $\begin{array}{l}\text { Correlimos } \\
\text { semipalmeado }\end{array}$ & INR & c \\
\hline & & Calidris mauri & Correlimos picudo & INR & c \\
\hline & & Calidris minutilla & Correlimos diminuto & INR & c \\
\hline & & Calidris bairdii & Correlimos patinegro & INR & $\mathrm{r}$ \\
\hline & & Calidris fuscicollis* & Correlimos rabiblanco & INR & $\mathrm{r}$ \\
\hline & & Calidris melanotos & Correlimos pectoral & INR & $\mathrm{r}$ \\
\hline & & Calidris himantopus* & Correlimos zancón & INR & $\mathrm{r}$ \\
\hline & Jacanidae & Jacana jacana & Gallito de ciénaga & $\mathrm{R}$ & c \\
\hline & Stercorariidae & Stercorarius parasiticus & Págalo parasítico & INR & c \\
\hline & \multirow{11}{*}{ Laridae } & Leucophaeus atricilla & Gaviota reidora & INR & c \\
\hline & & Anous stolidus* & Tiñosa común & ML & $\mathrm{r}$ \\
\hline & & Sternula antillarum ${ }^{*}$ & Gaviotín enano & IPR & $\mathrm{r}$ \\
\hline & & Sternula superciliaris & Gaviotín fluvial & $\mathrm{R}$ & $\mathrm{r}$ \\
\hline & & Phaetusa simplex & Gaviotín picudo & $\mathrm{R}$ & $\mathrm{r}$ \\
\hline & & Gelochelidon nilotica & Gaviotín blanco & IPR & $\mathrm{r}$ \\
\hline & & Hydroprogne caspia & Gaviotín piquirrojo & INR & $\mathrm{r}$ \\
\hline & & Chlidonias niger & Gaviotín negro & INR & $\mathrm{r}$ \\
\hline & & Sterna hirundo & Gaviotín común & INR & $\mathrm{r}$ \\
\hline & & Thalasseus sandvicensis & Gaviotín patinegro & INR & $\mathrm{r}$ \\
\hline & & Thalasseus maximus & Gaviotín real & INR & c \\
\hline & Rynchopidae & Rynchops niger & Rayador & IPR & $\mathrm{r}$ \\
\hline Cuculiformes & Cuculidae & Crotophaga major & Garrapatero mayor & $\mathrm{R}$ & c \\
\hline
\end{tabular}




\begin{tabular}{|c|c|c|c|c|c|}
\hline Orden & Familia & Especie & Nombre común & Estatus & Frecuencia \\
\hline \multirow{5}{*}{ Coraciiformes } & \multirow{5}{*}{ Alcedinidae } & Megaceryle torquata & Martín pescador mayor & $\mathrm{R}$ & c \\
\hline & & Chloroceryle amazona & $\begin{array}{l}\text { Martín pescador } \\
\text { matraquero }\end{array}$ & $\mathrm{R}$ & c \\
\hline & & Chloroceryle americana & Martín pescador chico & $\mathrm{R}$ & c \\
\hline & & Chloroceryle inda & $\begin{array}{l}\text { Martín pescador } \\
\text { selvático }\end{array}$ & $\mathrm{R}$ & $\mathrm{r}$ \\
\hline & & Chloroceryle aenea & $\begin{array}{l}\text { Martín pescador } \\
\text { pigmeo }\end{array}$ & $\mathrm{R}$ & c \\
\hline \multirow{8}{*}{ Passeriformes } & Furnariidae & $\begin{array}{l}\text { Certhiaxis } \\
\text { cinnamomeus }\end{array}$ & Rastrojero barbiamarillo & $\mathrm{R}$ & c \\
\hline & \multirow{3}{*}{ Tyrannidae } & Pitangus lictor & Bichofué menor & $\mathrm{R}$ & $\mathrm{r}$ \\
\hline & & Fluvicola pica & Viudita común & $\mathrm{R}$ & c \\
\hline & & $\begin{array}{l}\text { Arundinicola } \\
\text { leucocephala }\end{array}$ & Monjita pantanera & $\mathrm{R}$ & $\mathrm{r}$ \\
\hline & Hirundinidae & Tachycineta albiventer & Golondrina aliblanca & $\mathrm{R}$ & c \\
\hline & \multirow{2}{*}{ Parulidae } & Conirostrum bicolor & Conirrostro manglero & $\mathrm{R}$ & c \\
\hline & & Parkesia noveboracensis & Reinita acuática & INR & c \\
\hline & Icteridae & $\begin{array}{l}\text { Chrysomus } \\
\text { icterocephalus }\end{array}$ & Turpial cabeciamarillo & $\mathrm{R}$ & c \\
\hline
\end{tabular}


Anexo 2. Abundancia, registros y evidencia reproductiva de las especies pertenecientes a los grupos funcionales.

Appendix 2. Abundance, records and breeding evidence of the species of the functional groups.

\begin{tabular}{|c|c|c|c|c|c|}
\hline Grupo & Especie & Abundancia & $\begin{array}{l}\text { Número de } \\
\text { registros }\end{array}$ & Evidencia & Fechas \\
\hline \multirow{25}{*}{ Vadeadoras } & Phoenicopterus ruber & 1 & 2 & & \\
\hline & Jabiru mycteria & 1 & 3 & & \\
\hline & Mycteria americana & 17 & 16 & & \\
\hline & Tigrisoma lineatum & 10 & 38 & & \\
\hline & Tigrisoma mexicanum & 4 & 32 & $\begin{array}{l}\text { Nidos y } \\
\text { pollos }\end{array}$ & De 2009 a 2016 \\
\hline & Agamia agami & 164 & 18 & $\begin{array}{l}\text { Nidos y } \\
\text { pollos }\end{array}$ & De septiembre a octubre \\
\hline & Cochlearius cochlearius & 64 & 12 & $\begin{array}{l}\text { Nidos y } \\
\text { pollos }\end{array}$ & De septiembre a octubre \\
\hline & Ixobrychus exilis & 2 & 2 & & \\
\hline & Nycticorax nycticorax & 33 & 45 & $\begin{array}{l}\text { Nidos y } \\
\text { pollos }\end{array}$ & De septiembre a octubre \\
\hline & Nyctanassa violacea & 41 & 48 & & \\
\hline & Butorides virescens & 11 & 77 & & \\
\hline & Butorides striata & 26 & 104 & 1 pollo & $8 / 11 / 2011$ \\
\hline & Bubulcus ibis & 239 & 66 & $\begin{array}{l}\text { Nidos y } \\
\text { pollos }\end{array}$ & De julio a diciembre \\
\hline & Ardea herodias & 3 & 28 & & \\
\hline & Ardea cocoi & 339 & 99 & $\begin{array}{l}\text { Nidos y } \\
\text { pollos }\end{array}$ & $9 / 05 / 2016$ \\
\hline & Ardea alba & 700 & 183 & $\begin{array}{l}\text { Nidos y } \\
\text { pollos }\end{array}$ & De julio a octubre \\
\hline & Pilherodius pileatus & 1 & 1 & & \\
\hline & Egretta tricolor & 209 & 140 & $\begin{array}{l}\text { Nidos y } \\
\text { pollos }\end{array}$ & De julio a octubre \\
\hline & Egretta rufescens & 1 & 3 & & \\
\hline & Egretta thula & 600 & 125 & $\begin{array}{l}\text { Nidos y } \\
\text { pollos }\end{array}$ & De mayo a diciembre \\
\hline & Egretta caerulea & 134 & 141 & & \\
\hline & Eudocimus albus & 945 & 141 & $\begin{array}{l}\text { Nidos y } \\
\text { pollos }\end{array}$ & De julio a noviembre \\
\hline & Plegadis falcinellus & 555 & 36 & & \\
\hline & Phimosus infuscatus & 87 & 84 & & \\
\hline & Platalea ajaja & 20 & 26 & & \\
\hline
\end{tabular}




\begin{tabular}{|c|c|c|c|c|c|}
\hline Grupo & Especie & Abundancia & $\begin{array}{l}\text { Número de } \\
\text { registros }\end{array}$ & Evidencia & Fechas \\
\hline \multirow{25}{*}{ Limícolas } & Vanellus chilensis & 27 & 83 & Pollos & De marzo a junio \\
\hline & Pluvialis squatarola & 5 & 44 & & \\
\hline & $\begin{array}{l}\text { Charadrius } \\
\text { semipalmatus }\end{array}$ & 26 & 53 & & \\
\hline & Charadrius wilsonia & 8 & 23 & $\begin{array}{l}\text { Nidos y } \\
\text { pollos }\end{array}$ & $14 / 05 / 2013,16 / 06 / 2014,19 / 05 / 2015$ \\
\hline & Charadrius collaris & 6 & 24 & $\begin{array}{l}\text { Nidos y } \\
\text { pollos }\end{array}$ & $\begin{array}{l}25 / 05 / 2008,13 / 03 / 2011 \\
30 / 03 / 2011,15 / 05 / 2011\end{array}$ \\
\hline & Himantopus mexicanus & 57 & 57 & & \\
\hline & Gallinago delicata & 1 & 2 & & \\
\hline & Limnodromus griseus & 130 & 23 & & \\
\hline & Numenius phaeopus & 7 & 52 & & \\
\hline & Actitis macularius & 41 & 96 & & \\
\hline & Tringa melanoleuca & 15 & 48 & & \\
\hline & Tringa flavipes & 94 & 52 & & \\
\hline & Tringa solitaria & 9 & 36 & & \\
\hline & Tringa semipalmata & 13 & 56 & & \\
\hline & Arenaria interpres & 16 & 30 & & \\
\hline & Calidris canutus & 1 & 1 & & \\
\hline & Calidris alba & 6 & 13 & & \\
\hline & Calidris pusilla & 26 & 0,69 & & \\
\hline & Calidris mauri & 121 & 26 & & \\
\hline & Calidris minutilla & 64 & 50 & & \\
\hline & Calidris bairdii & 1 & 1 & & \\
\hline & Calidris fuscicollis & 1 & 1 & & \\
\hline & Calidris melanotos & 2 & 2 & & \\
\hline & Calidris himantopus & 5 & 5 & & \\
\hline & Jacana jacana & 166 & 89 & $\begin{array}{l}\text { Pollos y } 1 \\
\text { cópula }\end{array}$ & De marzo a diciembre \\
\hline \multirow{4}{*}{$\begin{array}{l}\text { Marinas y } \\
\text { martinetes }\end{array}$} & Calonectris diomedea & 1 & 1 & & \\
\hline & Fregata magnificens & 39 & 67 & & \\
\hline & Sula leucogaster & 1 & 1 & & \\
\hline & $\begin{array}{l}\text { Phalacrocorax } \\
\text { brasilianus }\end{array}$ & 1283 & 133 & $\begin{array}{l}\text { Nidos e } \\
\text { indivi- } \\
\text { duos con } \\
\text { material }\end{array}$ & $\begin{array}{l}\text { 16/10/2010, 1/11/2011, 13/10/2015, } \\
6 / 07 / 2016\end{array}$ \\
\hline
\end{tabular}




\begin{tabular}{|c|c|c|c|c|c|}
\hline Grupo & Especie & Abundancia & $\begin{array}{l}\text { Número de } \\
\text { registros }\end{array}$ & Evidencia & Fechas \\
\hline \multirow{20}{*}{$\begin{array}{l}\text { Marinas y } \\
\text { martinetes }\end{array}$} & Anhinga anhinga & 5 & 6 & $\begin{array}{l}1 \text { hembra } \\
\text { en nido }\end{array}$ & $13 / 10 / 2015$ \\
\hline & Pelecanus occidentalis & 105 & 72 & & \\
\hline & Stercorarius parasiticus & 4 & 5 & & \\
\hline & Leucophaeus atricilla & 5 & 10 & & \\
\hline & Anous stolidus & 1 & 1 & & \\
\hline & Sternula antillarum & 3 & 1 & & \\
\hline & Sternula superciliaris & 14 & 11 & & \\
\hline & Phaetusa simplex & 29 & 11 & & \\
\hline & Gelochelidon nilotica & 83 & 13 & & \\
\hline & Hydroprogne caspia & 1 & 1 & & \\
\hline & Chlidonias niger & 20 & 1 & & \\
\hline & Sterna hirundo & 1 & 1 & & \\
\hline & $\begin{array}{l}\text { Thalasseus } \\
\text { sandvicensis }\end{array}$ & 43 & 11 & & \\
\hline & Thalasseus maximus & 173 & 74 & & \\
\hline & Rynchops niger & 2 & 2 & & \\
\hline & Megaceryle torquata & 9 & 153 & & \\
\hline & Chloroceryle amazona & 6 & 69 & 1 nido & $18 / 10 / 2010,10 / 11 / 2012$ \\
\hline & Chloroceryle americana & 4 & 44 & & \\
\hline & Chloroceryle inda & 3 & 2 & & \\
\hline & Chloroceryle aenea & 3 & 33 & & \\
\hline \multirow{9}{*}{$\begin{array}{l}\text { Patos y } \\
\text { zambulli- } \\
\text { dores }\end{array}$} & Dendrocygna bicolor & 395 & 32 & & \\
\hline & Dendrocygna viduata & 159 & 30 & & \\
\hline & $\begin{array}{l}\text { Dendrocygna } \\
\text { autumnalis }\end{array}$ & 1118 & 88 & 4 pollos & $30 / 10 / 2009$ \\
\hline & Cairina moschata & 7 & 8 & & \\
\hline & Spatula discors & 387 & 69 & & \\
\hline & Spatula clypeata & 2 & 2 & & \\
\hline & Nomonyx dominicus & 4 & 1 & & \\
\hline & Tachybaptus dominicus & 36 & 15 & & \\
\hline & Podilymbus podiceps & 14 & 8 & & \\
\hline
\end{tabular}




\begin{tabular}{|c|c|c|c|c|c|}
\hline Grupo & Especie & Abundancia & $\begin{array}{l}\text { Número de } \\
\text { registros }\end{array}$ & Evidencia & Fechas \\
\hline \multirow{9}{*}{ Paludícolas } & Chauna chavaria & 60 & 77 & 2 nidos & $15 / 11 / 2012,17 / 10 / 2015$ \\
\hline & Aramus guarauna & 27 & 75 & 2 pollos & $14 / 03 / 2011$ \\
\hline & Aramides cajaneus & 4 & 29 & & \\
\hline & Aramides axillaris & 1 & 1 & & \\
\hline & Laterallus albigularis & 7 & 19 & 1 pollo & $13 / 04 / 2012$ \\
\hline & Porzana flaviventer & 1 & 1 & & \\
\hline & Porzana carolina & 1 & 1 & & \\
\hline & Gallinula galeata & 29 & 32 & $\begin{array}{l}\text { Nido y } \\
\text { pollos }\end{array}$ & $8 / 05 / 2013,16 / 03 / 2011,18 / 12 / 2014$ \\
\hline & Porphyrio martinicus & 23 & 65 & $\begin{array}{l}\text { Nido y } \\
\text { pollos }\end{array}$ & $2 / 11 / 2011,30 / 12 / 2013$ \\
\hline \multirow{6}{*}{$\begin{array}{l}\text { Rapaces } \\
\text { acuáticas }\end{array}$} & Pandion haliaetus & 4 & 52 & & \\
\hline & Rostrhamus sociabilis & 11 & 44 & $\begin{array}{l}\text { Con } \\
\text { material }\end{array}$ & $3 / 10 / 2010$ \\
\hline & Helicolestes hamatus & 1 & 1 & & \\
\hline & $\begin{array}{l}\text { Buteogallus } \\
\text { anthracinus }\end{array}$ & 4 & 63 & 1 nido & $7 / 05 / 2013$ \\
\hline & Buteogallus urubitinga & 1 & 5 & $\begin{array}{l}1 \text { nido con } \\
1 \text { pollo }\end{array}$ & $8 / 05 / 2013$ \\
\hline & Busarellus nigricollis & 4 & 47 & & \\
\hline \multirow{9}{*}{ No estrictas } & Crotophaga major & 40 & 98 & $\begin{array}{l}\text { Con } \\
\text { material }\end{array}$ & $2 / 11 / 2012$ \\
\hline & $\begin{array}{l}\text { Certhiaxis } \\
\text { cinnamomeus }\end{array}$ & 8 & 62 & $\begin{array}{l}\text { Con } \\
\text { material }\end{array}$ & $12 / 04 / 2012,10 / 11 / 2012,8 / 05 / 2013$ \\
\hline & Pitangus lictor & 1 & 1 & & \\
\hline & Fluvicola pica & 12 & 99 & 1 nido & $17 / 04 / 2012$ \\
\hline & $\begin{array}{l}\text { Arundinicola } \\
\text { leucocephala }\end{array}$ & 2 & 5 & & \\
\hline & Tachycineta albiventer & 26 & 34 & & \\
\hline & Conirostrum bicolor & 9 & 40 & & \\
\hline & Parkesia noveboracensis & 7 & 39 & & \\
\hline & $\begin{array}{l}\text { Chrysomus } \\
\text { icterocephalus }\end{array}$ & 75 & 48 & & \\
\hline
\end{tabular}




\section{Carlos Ruiz-Guerra}

Asociación para el Estudio y Conservación de las Aves en Colombia-Calidris.

Cali, Colombia.

https:/ / orcid.org/0000-0002-8150-9516

cjruiz@calidris.org.co

Autor para correspondencia

\section{Yanira Cifuentes-Sarmiento}

Asociación para el Estudio y Conservación de las Aves en Colombia-Calidris.

Cali, Colombia.

https:/ / orcid.org/0000-0003-0776-0953

ycifuentes@calidris.org.co
Aves acuáticas de la cuenca baja del río Sinú, Córdoba, Caribe Colombiano

Citación del artículo: Ruíz-Guerra, C. \& CifuentesSarmiento, Y. (2021). Aves acuáticas de la cuenca baja del río Sinú, Córdoba, Caribe Colombiano. Biota Colombiana, 22(2), 88-107.

https:/ / doi.org/10.21068/c2021.v22n02a055

Recibido: 14 de septiembre 2020

Aceptado: 4 de marzo 2021 